\title{
Quantitative electrophysiological study of alcoholic neuropathy
}

\author{
J OH N P A LLANTYNE, STIG HANSEN, ANDREW WEIR, \\ JOH R G WHITEHEAD, ANDPATRICK J M ULLIN
}

From the Glasgow University Department of Neurology and Division of Clinical Physics, Institute of Neurological Sciences and Department of Psychological Medicine, Southern General Hospital, Glasgow

SUMMARY Thirty-one chronic alcoholic patients were investigated using quantitative electrophysiological techniques. Estimates of the numbers of functioning motor units in the extensor digitorum brevis muscles and measurements of the parameters of the potentials of these units are presented along with the values for motor nerve conduction velocities in the inneivating lateral popliteal nerves. Motor conduction velocities and sensory nerve action potential amplitudes were also measured in the ulnar nerves. The results and their inter-relationships lead us to conclude that the slowing of motor nerve conduction and reduction in sensory nerve action potential amplitudes in alcoholic neuropathy are a consequence of axon loss. We found no evidence of pathological slowing of conduction in surviving axons. Reinnervation by functioning motor axons is poor compared to a number of other neuropathic conditions. In our patients there was no evidence of preferential involvement of sensory axons. The results support a predominant axonal dysfunction in alcoholic neuropathy.

The pathological basis of alcoholic neuropathy remains controversial. Some electrophysiological and pathological studies have favoured a primary axonal disturbance ${ }^{1-5}$ while others support a predominant or concomitant demyelinative pathogenesis. ${ }^{6} 7$ There is little quantitative information concerning the extent and severity of denervation and compensatory reinnervation in the peripheral motor system in alcoholic neuropathy and such quantitative data as are available, refer to sensory nerve studies and appear to favour the earliest involvement of sensory axons in this neuropathy. ${ }^{5}$

We have applied our computer assisted technique for the estimation of the number of functioning motor units in the human extensor digitorum brevis muscle (EDB) ${ }^{8}$ and the derivation of the parameters of a sample of electrically evoked motor unit potentials (MUPs) ${ }^{9}$ from the same muscle in a group of patients with alcoholic neuropathy in an effort to quantify the changes in motor parameters in that condition. We have also

Address for reprint requests: Dr JP Ballantyne, Department of Neurology, Institute of Neurological Sciences, Southern General Hospital, Glasgow G51 4TF, Scotland.

Accepted 13 December 1979 measured the fastest motor nerve conduction velocities (FMNCVs) and the shortest distal motor latencies (SDMLs) in the innervating lateral popliteal nerve and FMNCVs, sensory nerve conduction velocities and potential amplitudes in an ulnar nerve in each patient. The relationships between these parameters and their significance for the pathophysiology of alcoholic neuropathy are discussed. Where appropriate comparisons are drawn with the results we have obtained in other neuromyopathies.

\section{Methods}

The composition and placement of the surface recording electrodes over the EDB muscle, the properties of the stimulating electiodes over the anterior tibial nerve at the ankle, and the details of the rate and strength of stimulation used to evoke motor unit potentials have been described. ${ }^{8}$ The amplification and display systems, the computer handling of data for the estimation of motor unit numbers in the EDB muscle, and the computer derivation of the parameters of the electrically evoked motor unit potentials have also been reported. ${ }^{89}$ 
Briefly, MUPs recorded from surface electrodes over the EDB muscle, are evoked sequentially by finely graded incremental stimulation of the anterior tibial nerve at the ankle. Recruitment of up to 15 motor units can be recognised by a combination of visual and computer analysis of the muscle action potential increments displayed on the oscilloscope screen. The first MUP is displayed in isolation on the oscilloscope; the potential of the second is incorporated in a compound muscle action potential containing motor unit potentials 1 and 2. As each new potential is added to the preceding one, the compound muscle action potential so constituted is stored in a computer memory (template). Template 1 contains MUP 1, template 2 contains the sum of MUPs 1 and 2, template 3 contains the sum of MUPs 1, 2, and 3, and so on. Up to 15 templates can be stored. The number of motor units in the EDB muscle is calculated from the formula: $\mathbf{M U N}=[\mathrm{A}(\mathrm{M}) / \mathrm{A}(\mathrm{N})] \times \mathrm{N}$ where MUN is motor unit number, $A(M)=$ the area of the supramaximally evoked muscle action potential and $A(N)=$ the area of the compound muscle action potential containing $\mathbf{N}$ MUPs.

By a process of template subtraction, the computer also displays the first and sequentially recruited MUPs in isolation. For example, subtraction of template 1 from template 2 will leave MUP 2 in isolation, subtraction of template 2 from 3 will leave MUP 3 in isolation, and so on. The latencies, durations, amplitudes and areas of individual MUPs are then measured. Amplitudes and areas are provided by the computer while latencies and durations are measured manually from a computer printout. 9 All potential recordings are from surface electrodes over the EDB muscle.

The FMNCVs in the lateral popliteal nerve (knee to ankle segment) and SDMLs (anterior tibial nerve at the ankle to EDB muscle) were also measured from the same surface electrodes over the EDB muscle. The FMNCVs and SDMLs were also measured in one ulnar nerve in the below elbow to wrist segment. The motor response was recorded from silver strip electrodes over the hypothenar eminence as used over the EDB muscle. The stimulating electrode had similar properties to that used to stimulate the lateral popliteal nerve. Sensory nerve action potentials (SNAPs) evoked by stimulation of the little finger were recorded orthodromically over the ulnar nerve at the wrist from the gauze padded, saline soaked electrodes used for motor stimulation.

All investigations were undertaken in a thermostatically controlled room and limb temperature was maintained at $33^{\circ} \mathrm{C} \pm 1^{\circ} \mathrm{C}$.
Patients and control subjects

Thirty-one chronic alcoholic patients (mean age $47 \cdot 7 \pm 9.8$ years) were referred from the Department of Psychological Medicine at the Southern General Hospital with a tentative diagnosis of alcoholic peripheral neuropathy.

The presence of clinical neuropathy was subsequently evaluated by one of us (JPB) in terms of both the peripheral sensory and motor sytsems. Persistent symmetrical paraesthesiae, hypoalgesia or hyperalgesia with or without objective signs of sensory impairment were taken to indicate the presence of sensory neuropathy. Transient sensory disturbances lasting minutes and often present in the more introspective patients were discounted as evidence of neuropathy. A symmetrical reduction in power with or without wasting in the distal aspects of the legs along with a reduction or absence of tendon reflexes was considered objective evidence of motor involvement. On this basis, 22 of the 31 patients had symptoms or signs of a symmetrical peripheral polyneuropathy. So far as was possible, patients were excluded from this study who were thought to suffer from other disorders which might give rise to a peripheral neuropathy.

There were 27 control subjects (mean age 46.9土 12.4 years) (table 1). All control subjects were free of neurological disease and were drawn from the staff and their relatives of the Institute of Neurological Sciences, Glasgow.

Table 1 Age, shortest distal motor latency (SDML), fastest motor nerve conduction velocity (FMNCV) and motor unit numbers (MUN) in control subjects and patients with alcoholic neuropathy

\begin{tabular}{|c|c|c|c|c|}
\hline & $n$ & Mean & $S D$ & $p$ \\
\hline $\begin{array}{l}\text { Age (year) } \\
\text { Control } \\
\text { Alcoholic neur. } \\
\text { Sympt. group } \\
\text { Asympt. group }\end{array}$ & $\begin{array}{r}27 \\
31 \\
22 \\
9\end{array}$ & $\begin{array}{l}46 \cdot 9 \\
47 \cdot 7 \\
48 \cdot 2 \\
46 \cdot 2\end{array}$ & $\begin{array}{r}12 \cdot 4 \\
9 \cdot 8 \\
9 \cdot 6 \\
10 \cdot 7\end{array}$ & $\begin{array}{l}- \\
\text { NS } \\
\text { NS } \\
\text { NS }\end{array}$ \\
\hline $\begin{array}{l}\text { SDML (ms) } \\
\text { Control } \\
\text { Alcoholic neur. } \\
\text { Sympt. group } \\
\text { Asympt. group }\end{array}$ & $\begin{array}{r}27 \\
31 \\
22 \\
9\end{array}$ & $\begin{array}{l}3 \cdot 56 \\
4 \cdot 24 \\
4 \cdot 51 \\
3 \cdot 56\end{array}$ & $\begin{array}{l}0.45 \\
1.08 \\
1.14 \\
0.44\end{array}$ & $\begin{array}{l}<\overline{0.005} \\
<0.002 \\
\quad \text { NS }\end{array}$ \\
\hline $\begin{array}{l}\text { FMNCV (m/s) } \\
\text { Control } \\
\text { Alcoholic neur. } \\
\text { Sympt. group } \\
\text { Asympt. group }\end{array}$ & $\begin{array}{r}16 \\
31 \\
22 \\
9\end{array}$ & $\begin{array}{l}50 \cdot 5 \\
44 \cdot 8 \\
43 \cdot 9 \\
47 \cdot 1\end{array}$ & $\begin{array}{l}4 \cdot 59 \\
3 \cdot 97 \\
4 \cdot 04 \\
2 \cdot 80\end{array}$ & $\begin{array}{l}<\overline{0.0002} \\
<0.0002 \\
\quad \text { NS }\end{array}$ \\
\hline $\begin{array}{l}\text { MUN } \\
\text { Control } \\
\text { Alcoholic neur. } \\
\text { Sympt. group } \\
\text { Asympt. group }\end{array}$ & $\begin{array}{r}26 \\
31 \\
22 \\
9\end{array}$ & $\begin{array}{r}196 \cdot 2 \\
110 \cdot 2 \\
75 \cdot 5 \\
195 \cdot 1\end{array}$ & $\begin{array}{l}54 \cdot 3 \\
92 \cdot 6 \\
68 \cdot 6 \\
91 \cdot 5\end{array}$ & $\begin{array}{l}<\overline{0.0005} \\
<0.0001 \\
\text { NS }\end{array}$ \\
\hline
\end{tabular}




\section{Results}

All results are expressed as the mean \pm 1 standard deviation (SD) unless otherwise indicated. The statistical significances of the results were evaluated using Student's $t$ test.

LATERAL POPLITEAL NERVE STUDIES:

Shortest distal motor latencies There was a significant increase in the mean shortest distal motor latency (SDML) in the alcoholic patients compared to control subjects. The SDMLs had a significant negative correlation with the fastest motor nerve conduction velocities (FMNCVs) and a significant negative correlation with motor unit numbers (MUN) (table 3). In the asymptomatic group of alcoholic patients, the mean SDML was not different from the control value.

Fastest motor nerve conduction velocities The mean FMNCV was significantly reduced in the patients with clinical evidence of neuropathy compared to control subjects. In the asymptomatic alcoholic patients, however, the mean fastest motor nerve conduction velocity was not significantly different from the control value. The FMNCVs showed a significant positive correlation with MUNs and a significant negative correlation with SDMLs (table 3).

Motor unit numbers Mean MUNs were significantly reduced in the alcoholic patients with evidence of peripheral neuropathy. In the patients without evidence of peripheral neuropathy, mean MUNs were similar to control values (table 1).

MOTOR UNIT POTENTIAL PARAMETERS IN THE EXTENSOR DIGITORUM BREVIS MUSCLE

Motor unit potential latencies Mean motor unit potential (MUP) latencies were significantly prolonged in both the symptomatic and asymptomatic group of patients compared to control subjects (table 2). The mean MUP latency in each patient had a significant positive correlation with SDML and a significant negative correlation with FMNCV (table 3).

Motor unit potential durations in neither the symptomatic nor asymptomatic group of patients was mean MUP duration significantly different from control values (table 2 ).

Motor unit potential amplitudes In the symptomatic group of patients mean MUP amplitude was significantly increased over the control value (table 2). Mean MUP amplitude in the asymptomatic group was not significantly different from the control value (table 2 ).
Table 2 Motor unit potential (MUP) latency, duration, amplitude and area in control subjects and patients with alcoholic neuropathy

\begin{tabular}{lrrrr}
\hline & $n$ & Mean & $S D$ & $p$ \\
\hline MUP latency (ms) & & & & \\
Control & 150 & 4.63 & 0.98 & - \\
Alcoholic neur. & 200 & 5.36 & 1.37 & $<0.0001$ \\
Sympt. group & 132 & 5.50 & 1.44 & $<0.0001$ \\
Asympt. group & 68 & 5.09 & 1.20 & $<0.01$ \\
MUP duration (ms) & & & & \\
Control & 150 & 9.43 & 1.71 & - \\
Alcoholic neur. & 200 & 9.47 & 2.04 & $\mathrm{NS}$ \\
Sympt. group & 132 & 9.53 & 1.70 & $\mathrm{NS}$ \\
Asympt. group & 68 & 9.37 & 2.58 & $\mathrm{NS}$ \\
MUP amplitude ( $\mu \mathrm{V})$ & & & & \\
Control & 150 & 61.9 & 30.1 & - \\
Alcoholic neur. & 200 & 76.6 & 65.2 & $<0.01$ \\
Sympt. group & 132 & 80.4 & 73.8 & $<0.01$ \\
Asympt. group & 68 & 69.2 & 43.2 & $\mathrm{NS}$ \\
MUP area (arbitrary units) & & & & \\
Control & 150 & 17.4 & 8.4 & - \\
Alcoholic neur. & 200 & 21.3 & 16.8 & $<0.005$ \\
Sympt. group & 132 & 22.6 & 19.3 & $<0.005$ \\
Asympt. group & 68 & 18.8 & 10.1 & $\mathrm{NS}$ \\
\hline
\end{tabular}

Motor unit potential areas In the symptomatic patients mean MUP area was significantly increased compared to the mean control value while in the asymptomatic patients there was no significant difference from the normal mean value (table 2).

Ulnar nerve studies The mean FMNCV and the mean sensory nerve conduction velocity were not significantly different from controls.

Ten of the 31 patients had sensory nerve action potential (SNAP) amplitudes within the normal range (mean $9.8 \pm 3.9 \mu \mathrm{V}$ ) but 15 patients had MUNs within the normal range. Eight patients with normal SNAP amplitudes had normal MUNs. There was a significant positive correlation between MUNs in the EDB muscle and SNAP amplitudes in the ulnar nerve (table 3 ).

\section{Discussion}

We have found in the past that where there is pathological slowing of conduction velocity in intramuscular nerve twigs of the motor unit, a prolongation of the surface recorded MUP

Table 3 Electrophysiological correlations referred to in text: patients with alcoholic neuropathy

\begin{tabular}{llll}
\hline & & $r$ & $p$ \\
\hline SDML & $\sim$ FMNCV & -0.495 & $<0.01$ \\
SDML & $\sim$ MUN & -0.607 & $<0.001$ \\
FMNCV & $\sim$ MUN & +0.465 & $<0.01$ \\
Mean MUP latency & $\sim$ SDML & +0.770 & $<0.001$ \\
Mean MUP latency & $\sim$ FMNCV & -0.445 & $<0.02$ \\
MUN & $\sim$ Ulnar SNAP amplitude & +0.512 & $<0.01$ \\
\hline
\end{tabular}


occurs. This is a consequence of the accentuation in the differences in conduction time through the longest and shortest intramuscular nerve twigs of the unit. ${ }^{1011}$ We have noted a prolongation of the MUP duration in those conditions in which segmental demyelination is an undisputed histological feature of the neuropathy, as in the GuillainBarré syndrome ${ }^{12}$ and diabetic neuropathy. ${ }^{11}$ However, in renal neuropathy which histologically shows significant axonal changes and in motor neurone disease, where there is considerable loss of anterior horn cells, the MUP is of normal or short duration. ${ }^{13}{ }^{14}$ Similarly in the present study of alcoholic neuropathy, the mean MUP duration is not different from the control value. This result suggests that pathological slowing of conduction does not occur in the intramuscular nerve twigs of the motor unit and conversely, that pathological slowing of conduction is not present in the viable motor axons of the unit. ${ }^{14}$ In both diabetic neuropathy ${ }^{11}$ and the Guillain-Barre neuropathy, ${ }^{12}$ there is a significant positive correlation between MUP durations and either SDMLs or MUP latencies, further confirming the contribution of slowing of conduction velocity in the intramuscular nerve twigs to the increase in duration of the motor unit potential. Such a correlation is absent in alcoholic neuropathy and in motor neurone disease. ${ }^{14} \mathrm{We}$ have noted, however, that in alcoholic neuropathy there is a significant positive correlation between FMNCVs and MUNs (table 3) indicating that loss of motor axons plays a significant part in the reduction of the FMNCV in this condition. Despite this correlation FMNCVs are relatively well maintained in the presence of marked loss of motor units (fig 1). Only two patients had motor nerve conduction velocities below $40 \mathrm{~m} / \mathrm{s}$ and these remained within the range of physiological values for smaller motor axons. ${ }^{15}$ Three patients with very low MUNs (2, 5 and 6) had FMNCVs of 40, 41 and $43 \mathrm{~m} / \mathrm{s}$ respectively. These results suggest that a preferential loss of fast conducting large diameter motor axons does not occur in alcoholic neuropathy. The proportionality between conduction velocity values and MUNs would, however, be compatible with the hypothesis that there is a random loss of fast conducting large diameter axons in a process of random loss of axons of all sizes. The viable motor axons in alcoholic neuropathy are conducting at physiological rates. We have come to a similar conclusion in motor neurone disease. ${ }^{14}$ In that circumstance we would anticipate comparable reductions in motor nerve conduction velocities for comparable falls in motor unit numbers in these two conditions. This is the case as illustrated in fig 2. Fastest motor nerve conduction velocities show a significant negative correlation to SDMLs (table 3). The prolongation of SDML in alcoholic neuropathy will also be a consequence of the loss of fastest conducting axons as part of the process of random loss of motor axons.

There is a significant reduction in the numbers of functioning motor units in alcoholic neuropathy (table 1) and the mean area of the potentials of surviving motor units is significantly increased compared to the control value (table 2). This increase in MUP area, which we have considered

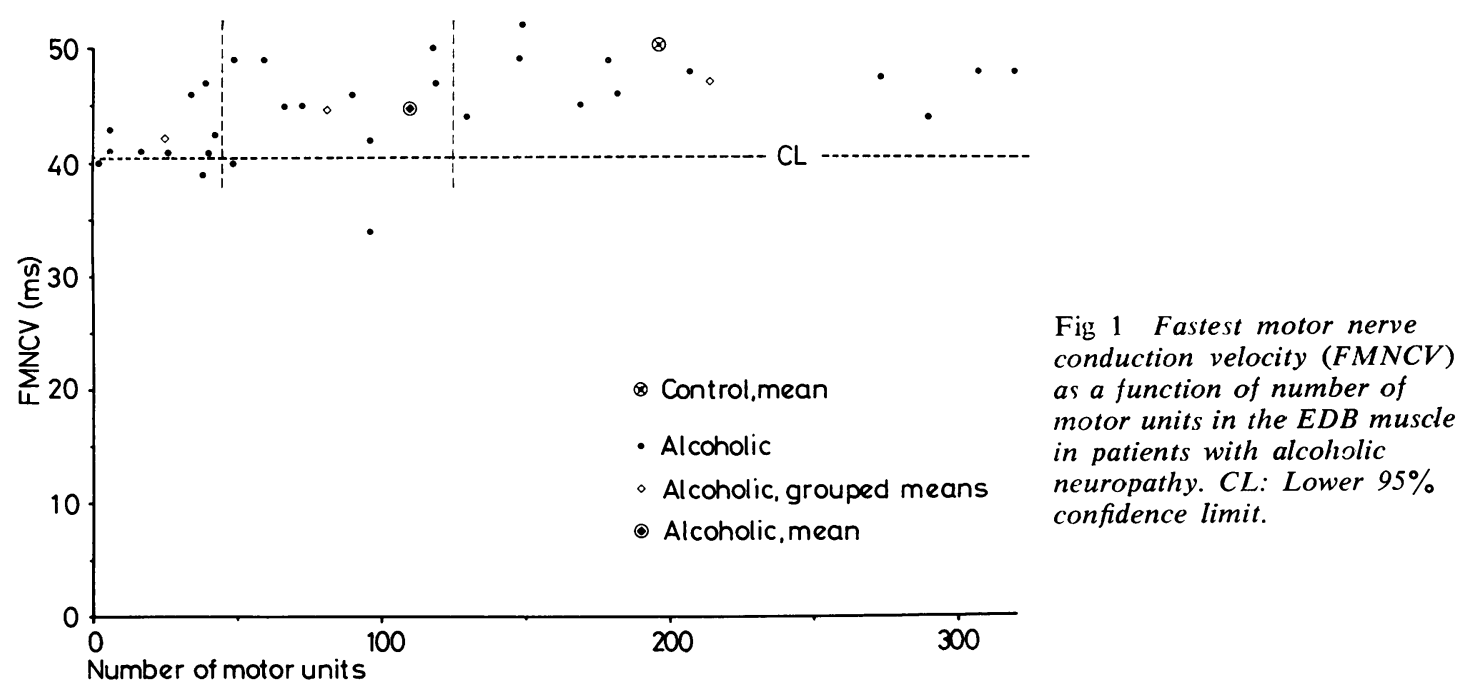

Fig 1 Fastest motor nerve conduction velocity (FMNCV) as a function of number of motor units in the EDB muscle in patients with alcoholic neuropathy. CL: Lower 95\% confidence limit. 


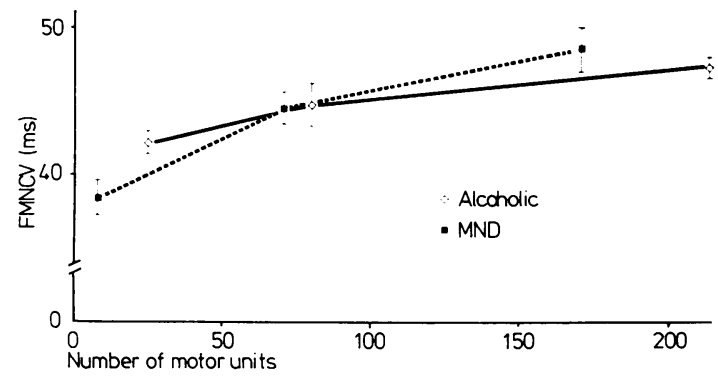

Fig 2 Fastest motor nerve conduction velocity (grouped means $\pm S E M$ ) as a function of mean number of motor units in patients with alcoholic neuropathy and motor neurone disease.

an index of reinnervation by surviving units ${ }^{10}$ is, however, relatively small. It is less than the increase in mean MUP area we have found in other denervating conditions (table 4) particularly motor

Table 4 Mean motor unit potential area in some neuropathies and motor neurone disease

\begin{tabular}{llll}
\hline & $n$ & $\begin{array}{l}\text { Mean } \\
\text { MUP area }\end{array}$ & $S D$ \\
\hline Alcoholic neuropathy & 200 & $21 \cdot 3$ & $16 \cdot 8$ \\
Diabetic neuropathy & 270 & $32 \cdot 1$ & $35 \cdot 9$ \\
Guillain-Barré neuropathy & 117 & $24 \cdot 6$ & $22 \cdot 0$ \\
Motor neurone disease & 200 & $42 \cdot 2$ & $43 \cdot 0$ \\
\hline
\end{tabular}

neurone disease ${ }^{14}$ (see fig 3), diabetic neuropathy ${ }^{11}$ and Guillain-Barré syndrome. ${ }^{12}$

There is, therefore, evidence of a diffuse derangement of function in the form of a reduced reinnervating capacity affecting most, if not all, of the motor axons in alcoholic neuropathy.

It has been claimed that sensory nerve fibres are involved at an earlier stage than motor nerve fibres in alcoholic neuropathy. ${ }^{5}$ We have examined our data in the light of that hypothesis. Electrophysiologically 10 patients had normal SNAP amplitudes in the ulnar nerves while 15 had normal MUNs in the EDB muscles (not significantly different by Chi square test). In our patients therefore, there is no evidence of preferential involvement of sensory relative to motor fibres.

Pathological slowing of conduction could lead to a diminution of the sensory nerve action potential amplitude by dispersion of the component single-fibre potentials but would not lead to a reduction in the numbers of motor units estimated in the EDB muscle by our technique. ${ }^{8}$ If we can assume qualitatively similar pathophysiological processes affecting motor and sensory axons then the significant positive correlation between motor unit numbers in the EDB muscle and SNAP amplitudes in the ulnar nerve (table 3 ) is not compatible with pathological slowing of conduction but indicates loss of both motor and sensory axons in the respective nerve trunks.

\section{Conchusions}

In terms of our results, the slowing of fastest motor nerve conduction velocity in alcoholic neuropathy is a consequence of the reduction in the number of functioning motor axons but those axons that remain viable are conducting at physiological rates. We have found no evidence of pathological slowing of conduction velocity. Despite the considerable reduction in the numbers of functioning motor units in the EDB muscles of these patients, reinnervation by the surviving units is relatively poor, indicating a diffuse and widespread disturbance of axonal function. We found no electrophysiological evidence of preferential involvement sensory nerve fibres.

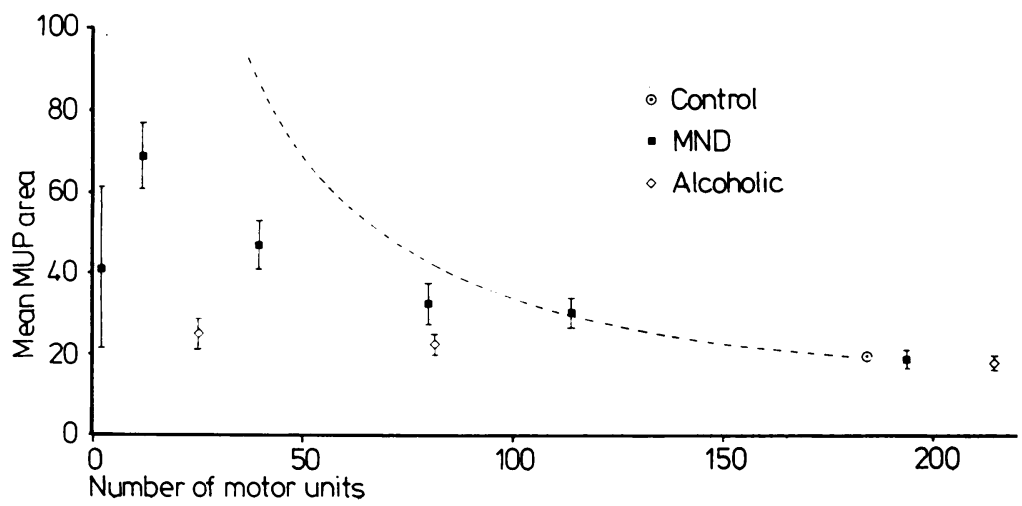

Fig 3 Motor unit potential area (grouped means $\pm S E M$ ) as a function of mean number of motor units in patients with alcoholic neuropathy and motor neurone disease. The broken line represents theoretical values for $100 \%$ reinnervation. Number of motor units 
Our electrophysiological studies favour the presence of a predominantly axonal disturbance in alcoholic neuropathy with loss of motor and sensory axons as the disease progresses.

We thank Professor JA Simpson for his help and encouragement and Mrs N Murdoch for secretarial assistance. This investigation was supported by a grant to AW from the Muscular Dystrophy Group of Great Britain.

\section{References}

1 Gilliat RW, Sears TA. Sensory nerve action potentials in patients with peripheral nerve lesions. J Neurol Neurosurg Psychiatry 1958; 21: 109-18.

2 Walsh JC, McLeod JF. Alcoholic neuropathy: an electrophysiological and histological study. $J$ Neurol Sci 1970; 10:457-69.

3 Blackstock E, Rushworth G, Gath D. Electrophysiological studies in alcoholism. J Neurol Neurosurg Psychiatry 1972; 35:326-34.

4 Tredici G, Minazzi M. Alcoholic neuropathy. An electron-microscopic study. J Neurol Sci 1975; 25:333-46.

5 Behse F, Buchthal F. Alcoholic neuropathy: Clinical, elecrophysiological and biopsy findings. Ann Neurol 1977; 2:95-100.

6 Mawdsley C, Mayer RF. Nerve conduction in alcoholic polyneuropathy. Brain 1965; 88:335-56.

7 Willer JC, Dehen H. Respective importance of different electrophysiological parameters in alco- holic neuropathy. J Neurol Sci 1977; 33: 387-96.

8 Ballantyne JP, Hansen S. A new method for the estimation of the number of motor units in a muscle. I. Control subjects and patients with myasthenia gravis. J Neurol Neurosurg Psychiatry 1974; 37:907-15.

9 Ballantyne JP, Hansen S. Computer method for the analysis of evoked motor unit potentials. I. Control subjects and patients with myasthenia gravis. J Neurol Neurosurg Psychiatry 1974; 37: 1195-201.

10 Ballantyne JP, Hansen S. Computer method for the analysis of evoked motor unit potentials. 2. Duchenne, limb-girdle, facioscapulohumeral and myotonic muscular dystrophies. J Neurol Neurosurg Psychiatry 1975; 38:417-28.

11 Hansen S, Ballantyne JP. Axonal dysfunction in the neuropathy of diabetes mellitus. A quantitative electrophysiological study. J Neurol Neurosurg Psychiatry 1977; 40:555-64.

12 Martinez-Figueroa A, Hansen S, Ballantyne JP. A quantitative electrophysiological study of acute idiopathic polyneuritis. J Neurol Neurosurg Psychiatry 1977; 40:156-61.

13 Hansen S, Ballantyne JP. A quantitative electrophysiological study of uraemic neuropathy. Diabetic and renal neuropathies compared. $J$ Neurol Neurosurg Psychiatry 1978; 41:128-34.

14 Hansen S, Ballantyne JP. A quantitative electrophysiological study of motor neurone disease. $J$ Neurol Neurosurg Psychiatry 1978; 41:773-83.

15 Thomas PK, Sears TA, Gilliatt RW. The range of conduction velocities in normal motor nerve fibres to the small muscles of the hand and foot. J Neurol Neurosurg Psychiatry 1959; 22:175-81. 